\title{
Retinal image generation method for retinal projection type super multi-view 3D head-mounted display
}

\section{Junya Kohno, Kayo Yoshimoto, Hideya Takahashi}

\begin{tabular}{|c|l|}
\hline Citation & Proceedings Volume 11304, Advances in Display Technologies X; 1130413 \\
\hline Issue Date & 2020-02-26 \\
\hline Description & Event: SPIE OPTO, 2020, San Francisco, California, United States \\
\hline Type & Conference Paper \\
\hline Textversion & Author \\
\hline Rights & $\begin{array}{l}\text { C (2020) COPYRIGHT Society of Photo-Optical Instrumentation Engineers } \\
\text { (SPIE). One print or electronic copy may be made for personal use only. } \\
\text { publication for a fee or for commercial purposes, and modification of the contents } \\
\text { of the publication are prohibited. } \\
\text { After it is published, it will be found at https://doi.org/10.1117/12.2545393. }\end{array}$ \\
\hline DOI & 10.1117/12.2545393 \\
\hline
\end{tabular}

\author{
Self-Archiving by Author(s) \\ Placed on: Osaka City University
}

Junya Kohno, Kayo Yoshimoto, and Hideya Takahashi "Retinal image generation method for retinal projection type super multi-view 3D head-mounted display", Proc. SPIE 11304, Advances in Display Technologies X, 1130413 (26 February 2020); https://doi.org/10.1117/12.2545393 


\title{
Retinal image generation method for retinal projection type super multi-view 3D head-mounted display
}

\author{
Junya Kohno ${ }^{\text {a }}$, Kayo Yoshimoto ${ }^{\text {a }}$, and Hideya Takahashi ${ }^{* a}$ \\ aDept. of Electrical and Information Engineering, Graduate School of Engineering, Osaka City Univ., \\ 3-3-138 Sugimoto, Sumiyoshi-ku, Osaka 558-8585, Japan;
}

\begin{abstract}
We have proposed the retinal projection type super multi-view head mounted display (HMD). The super multi-view method provides stereoscopic information by inducing the accommodation of the human eye by blurring the retinal image. This method uses the principle of Maxwellian view and reconstruct the blur image by overlapping projected each parallax image on the retina. However, considering only the degree of the overlap of the projected parallax images on the retina is not enough for reconstructing the real blur. In order to overcome this problem, we propose the optimal method considering not only the degree of the overlap of the parallax images projected on the retina but also the light intensity of each parallax image to reconstruct the real blur. From the evaluation experiment, we verified that the proposed method improves the reproducibility of blur compared to the method considering only the degree of superimposition of the parallax images projected on the retina. Furthermore, it was also confirmed that the prototype system can display 3D images in the depth range of human ocular accommodation from $200 \mathrm{~mm}$ to $2000 \mathrm{~mm}$. The super multi-view HMD based on the proposed method can induce the accommodation of the human eye with the high reproducibility blur and provide more natural $3 \mathrm{D}$ images.
\end{abstract}

Keywords: retinal projection display, super multi-view, head-mounted display, 3D display

\section{INTRODUCTION}

It has attracted attention that augmented reality $(\mathrm{AR})$ and mixed reality $(\mathrm{MR})^{1}$ which fuse the real world and the virtual world is realized by using the see-through type head mounted displays (HMDs). Many of the previously developed see-through HMDs provided 2D images. In order to realize natural AR or MR, it is necessary to provide virtual images without discomfort together with the real world. However, it is difficult for $2 \mathrm{D}$ images to be associated with the 3D real world and it causes a sense of discomfort to the observer. Therefore, in order to superimpose virtual images on the real world without discomfort, it is necessary to display 3D virtual images. To overcome this problem, we have previously proposed a retinal projection type super multi-view $\mathrm{HMD}^{2-7}$. The super multi-view system ${ }^{8}$ induces the accommodation of the human eye, which is one of the factors of stereoscopic vision, and enables to display monocularly 3D images. The previously proposed retinal projection type super multi-view HMD uses the principle of Maxwellian view and induce the accommodation of the observer's eye by the blur image caused by the displacement of the retinal image position of the projected parallax images. In the previous method, we focused only on the degree of overlap of the projected parallax images on the retina to reconstruct the blur image, but it was not enough for reconstructing the real blur. In order to solve this problem, we propose the optimal method considering not only the degree of the overlap of the parallax images projected on the retina but also the light intensity of each parallax image to reconstruct the real blur. To verify the effectiveness of the proposed method, we constructed the prototype system and confirmed that the proposed method improves the reproducibility of blur compared to the previously proposed method. Furthermore, we confirmed that the prototype system can display $3 \mathrm{D}$ images in the depth range of human ocular accommodation from $200 \mathrm{~mm}$ to $2000 \mathrm{~mm}$. Therefore, it was verified that the super multi-view HMD based on the proposed method can reconstruct the retinal image close to the real blur in the range of human ocular accommodation.

*hideya@osaka-cu.ac.jp; 


\section{RETINAL PROJECTION TYPE SUPER MULTI-VIEW HMD}

In Section 2, the previously proposed see-through type retinal projection super multi-view HMD.

\subsection{Super multi-view}

When humans look at an object, it recognizes the object by observing the light reflected from the object. Furthermore, an infinite number of rays of the reflected light are incident on the observer's eye to occur the imaging relationship between the object and the image on its retina and it recognizes the object three-dimensionally by being caused the accommodation of its eye. Therefore, we consider discrete sampling reflected light from an object as multiple parallax rays as shown in Figure 1 (a). In this situation, the state in which two or more parallax rays always enter the eye is called "super multi-view state". In the super multi-view state, reconstructed 3D images have very smooth motion parallax, and if an observer focuses his or her eyes on the reconstructed object's surface, the stimulus for the accommodation of the human eye is induced naturally. Therefore, the super multi-view HMD provides the observer with natural 3D virtual images so long as the super multi-view condition is satisfied. In Figure 1(b), each pixel which reconstructs a ray is a corresponding point of each parallax image on the display panel. It is possible to freely set the depth position of the virtual 3D point (image) by changing the parallax of the parallax images as shown in Figures 1(b) and 1(c).

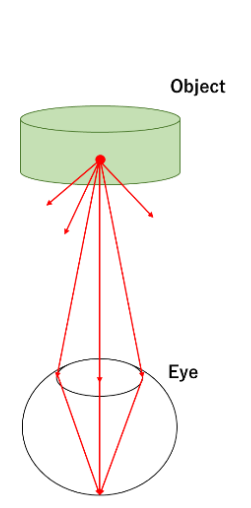

(a)

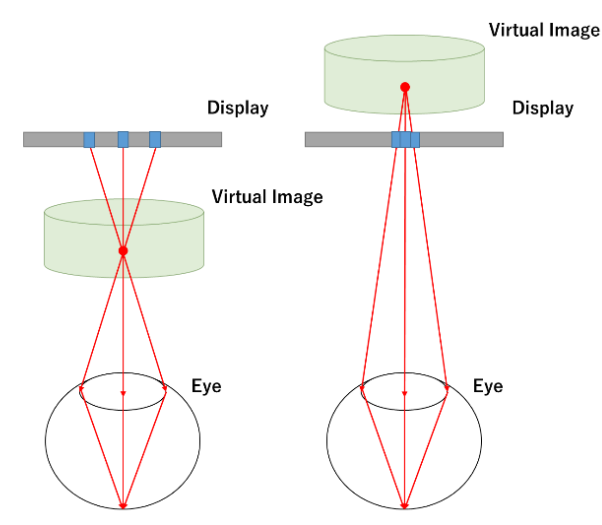

(b)

(c)

Figure 1. Schematic diagram of the stimulus of accommodation: (a) real world, (b)(c) super multi-view display

\subsection{Principle of super multi-view HMD}

As an image projection method, the super multi-view HMD uses the principle of Maxwellian view ${ }^{9}$. The principle of Maxwellian view is a technology that focuses a collimated image at the center of the pupil and projects it directly on the retina. As shown in Figure 2, the super multi-view state is realized by converging multiple parallax images to different positions on the pupil. For simplicity, we limit the number of parallax images to 3 . The parallax image \#i converges on the convergence point $\mathrm{Ci}$. Pi and Qi are parallax rays of the parallax image \#i which correspond to virtual 3D points $\mathrm{P}$ and $\mathrm{Q}$, respectively. As shown in Figure 3(a), when the observer's eye accommodates to the spatial position P, the projected images of the parallax rays P1, P2 and P3 are focused to the same position on the retina. The observer feels this image as the 3D point at the distance $L_{P}$. On the other hand, the projected images of the parallax rays Q1, Q2 and Q3 are focused to different positions on the retina. it feels this image as the blurred point at the distance $L_{Q}$. As shown in Figure 3(b), when its eye accommodates to the spatial position Q, the projected images of the parallax rays Q1, Q2 and Q3 are focused to the same position on the retina and the parallax rays P1, P2 and P3 are focused to different positions on the retina. The observer feels that the image of $Q$ is in focus and the image of $P$ is out of focus. Therefore, the super multi-view HMD can display the virtual image at the distance within the ability for focusing on the eye. 
HOE

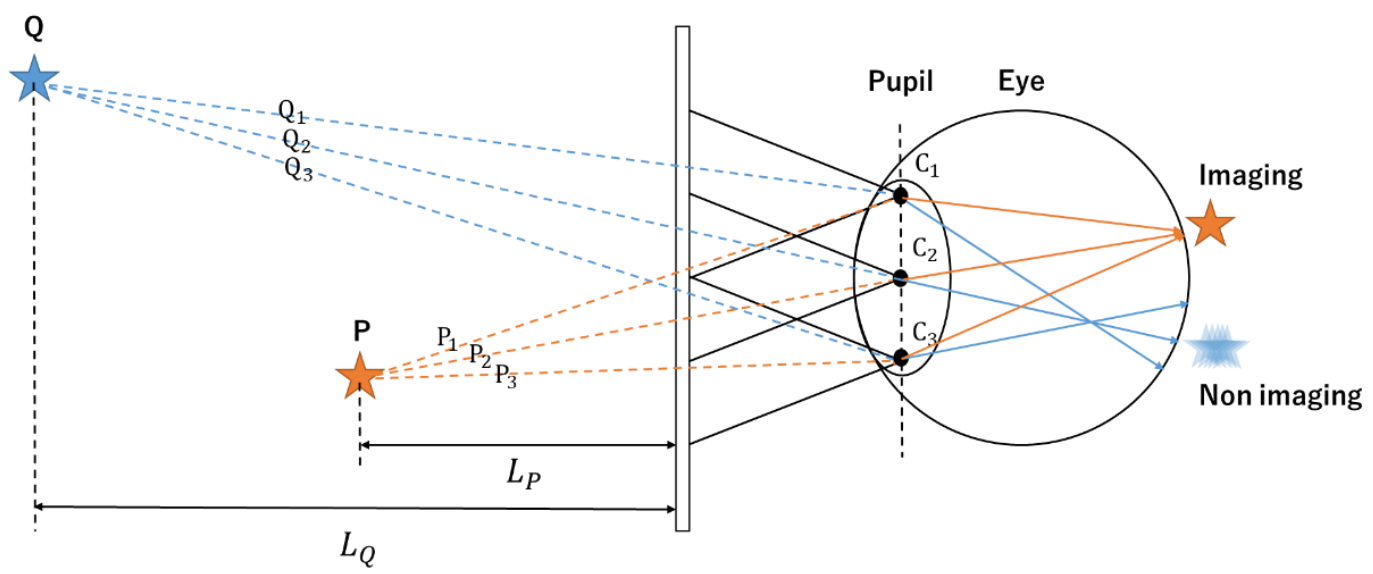

Figure 2. Schematic diagram of the retinal projection super multi-view HMD. The super multi-view condition is realized by some projected parallax images which converge on the different positions on the pupil.

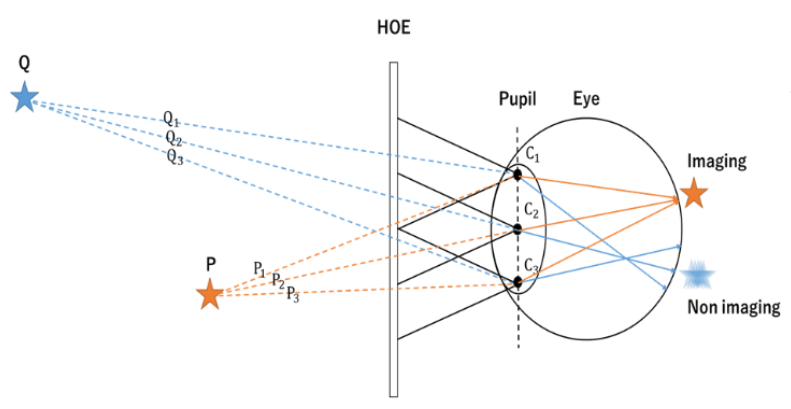

(a)

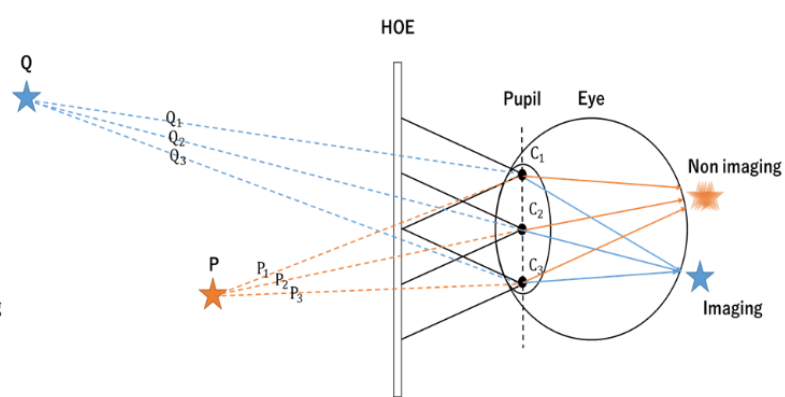

(b)

Figure 3. Principle of the 3D HMD by using the super multi-view method: (a) focusing on nearby point $P$, (b) focusing on far-off point Q.

\subsection{Previously proposed retinal projection type super multi-view HMD}

There are two types of methods for realizing a retinal projection type super multi-view HMD, a method ${ }^{2-4}$ of performing space division multiplex projection of parallax images and a method ${ }^{6}$ of performing time division multiplex projection of parallax images. Figure 4 shows the schematic diagram of the see-through retinal projection type super multi-view HMD by using the time division projection optical system. The proposed HMD consists of the multiple exposure holographic lens HOE2 which forms multiple convergence points, a high frame rate displaying device, a high-speed shutter, and a holographic lens which works as a converging lens and a combiner. In this HMD, multiple parallax images are displayed by time division, and these images are converged on respective points by the multiple exposure holographic lens. The optical shutter which synchronized to the display device passes only one convergence light corresponding to the correct parallax image. Therefore, proposed HMD realizes the super multi-view condition. 


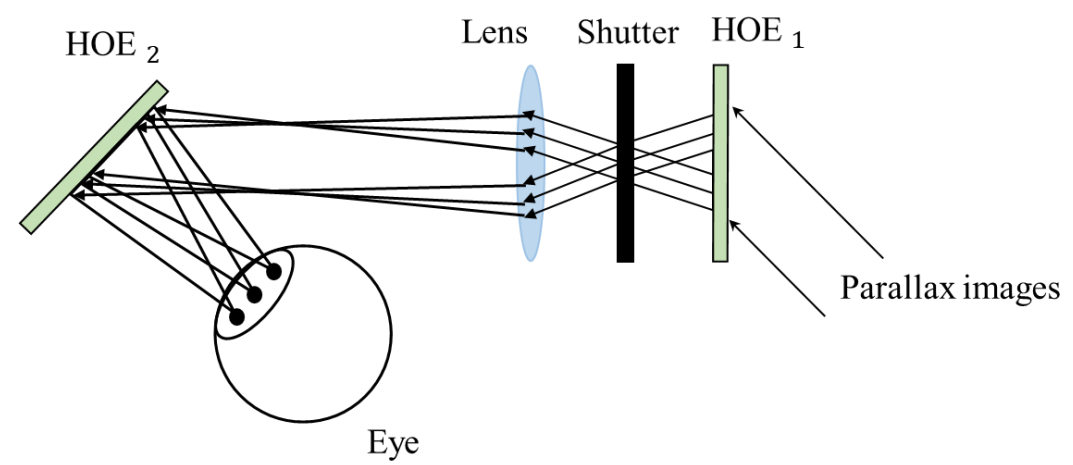

Figure 4. Schematic diagram of the retinal projection type super multi-view HMD by using the time division projection optical system.

\section{OPTIMAL METHOD FOR IMPROVING BLUR IMAGE REPRODUCIBILITY}

The retinal projection type super multi-view 3D display provides the observer with the 3D image by allowing them to observe the blur of image due to the overlap of multiple parallax images and induces the accommodation of the observer's eye. In this paper, we propose the improved method to reconstruct the blur image. In this method, by considering not only the degree of overlap of the parallax image but also the light intensity distribution of the parallax image, the blur image due to the overlap of the parallax image can be approximated to the real blur image. In order to improve the reproducibility of the blur image in the depth range of human ocular accommodation from $200 \mathrm{~mm}$ to $2000 \mathrm{~mm}$, we define the relationship between the number of parallax images and the light intensity of each parallax image and the retinal image, and derive the optimal system configuration for reconstructing the blur image.

\subsection{Position of the projected parallax images on the retina}

We derive positional displacement of the projected image on the retina by geometric optics. Figure 5 shows the schematic diagram of an optical system that projects a virtual point light source. Figure 6 shows the schematic diagram of the $\mathrm{x}-\mathrm{Z}$ plane of Figure 5. Table 1 shows the parameters used in Figure 6.

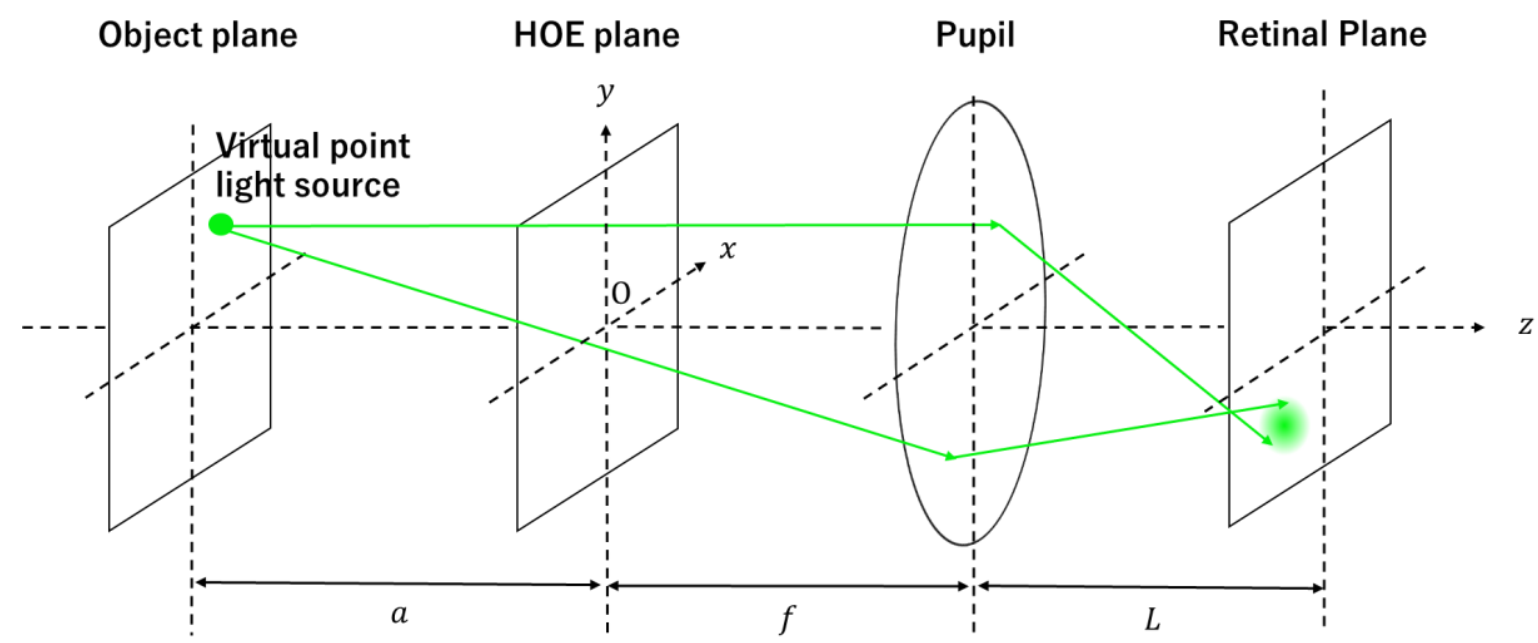

Figure 5. Schematic diagram of an optical system that projects a virtual point light source. 


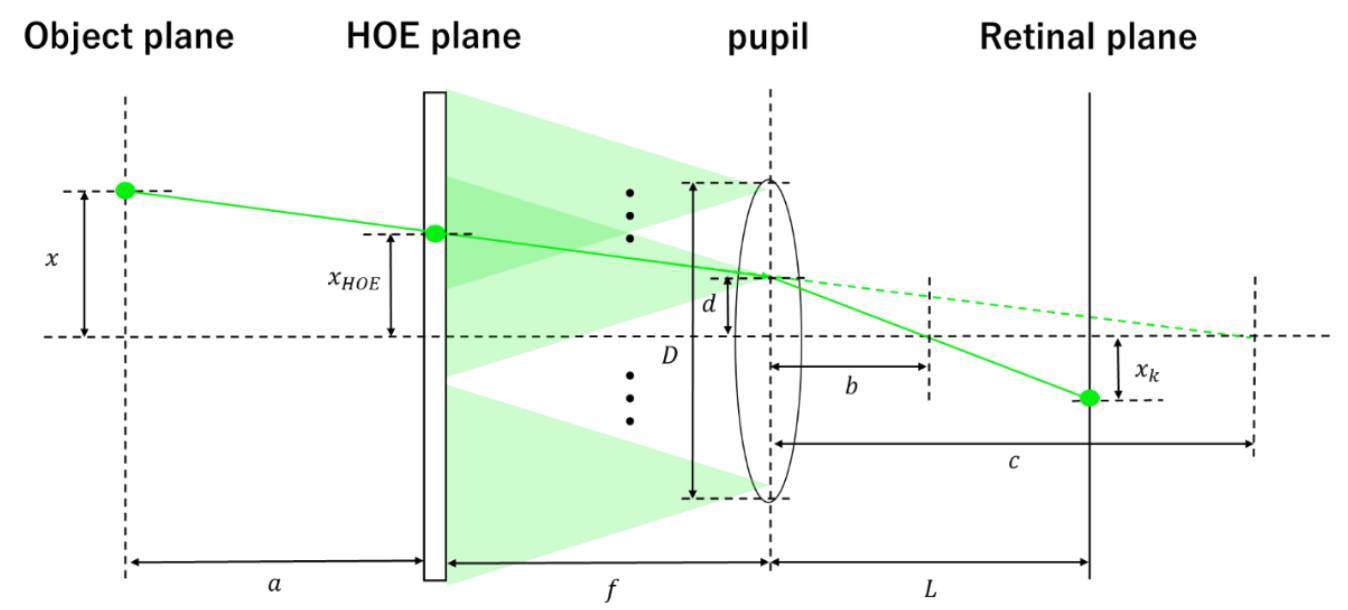

Figure 6. Schematic diagram of the $\mathrm{x}-\mathrm{z}$ plane of the optical system.

Table 1. Parameters in Figure 6.

\begin{tabular}{|c|c|}
\hline variable & meaning \\
\hline$x$ & x direction distance of the virtual point source \\
\hline$a$ & Distance from the virtual point source to the HOE \\
\hline$c$ & $\begin{array}{r}\text { Distance from the pupil to the intersection of the optical axis } \\
\text { and the ray before refracted }\end{array}$ \\
\hline$L$ & Distance from the pupil to the retina \\
\hline$f$ & HOE's focal length \\
\hline$f_{p}$ & Pupil's focal length \\
\hline$D$ & Pupil diameter \\
\hline$d$ & Distance from the optical axis to the k-th convergence point \\
\hline$x_{\mathrm{HOE}}$ & Distance from the optical axis to the ray on the HOE \\
\hline$x_{k}$ & The position of the projected image on the retina \\
\hline
\end{tabular}

We consider the position of the projected one pixel in the $k$-th parallax image on the retina when the super multi-view state as shown in Figure 5 is realized with superimposed projected $N$ parallax images. In Figure 6, from the lens formula and the geometric relationship, $x_{\mathrm{HOE}}, d, c$, and the position of the projected image on the retina $x_{k}$ can be obtained by

$$
\begin{gathered}
x_{H O E}=\frac{d-x}{a+f} a+x, \\
d=D \frac{N-2 k+1}{2(N-1)}, \\
\mathrm{c}=-\frac{a+f}{d-x} x,
\end{gathered}
$$

and

$$
x_{k}=d\left(1-\frac{f_{p}+c-f}{f_{p}(c-f)} L\right) \text {. }
$$




\subsection{Optimal light intensity of parallax images for reconstructing blur image}

The super multi-view 3D HMD enables displaying 3D images by allowing the observer to observe the blur image due to the overlap of each parallax image. Furthermore, by adjusting the light intensity of each parallax image, a blur image close to a real blur can be reconstructed. We consider that a virtual point light source is reconstructed by one pixel of parallax images. Figure 7 is the schematic diagram showing how a retinal image projected by the super multi-view method is formed. In Figure 7 and 8, the vertical axis in each graph represents the light intensity, and the horizontal axis represents the position on the retina. As shown in Figure 7, a retinal image projected by the super multi-view method is an image obtained by overlapping retinal images due to each parallax images, and the light intensity distribution $I_{\mathrm{smv}}\left(x_{k}, f_{p}, z\right)$ of the overlapped retinal image can be represented by the sum of $I_{\mathrm{s}}(x)$, which is the light intensity distribution of the projected one pixel of a parallax image on the retina. When retinal images due to unit pixels are overlapped and the number of parallax images is $N, I_{\mathrm{smv}}\left(x_{k}, f_{p}, z\right)$ is given by

$$
I_{s m v}\left(x, f_{p}, z\right)=\sum_{k=1}^{N} I_{s}\left(x-x_{k}\right) .
$$

Next, as shown in Figure 8, considering $I_{\mathrm{s}}(x)$ are weighted, and the light intensity $I_{\text {smv }}\left(x_{k}, f_{p}, z\right)$ obtained by overlapping weighted $I_{\mathrm{S}}(x)$. At the position $x_{k}$ which $k$-th parallax image is projected on the retina, when $I_{\text {smv }}^{\prime}\left(x_{k}, f_{p}, z\right)$ is made equal to the light intensity $I_{\text {real }}\left(x_{k}, f_{p}, z\right)$ of the retinal image due to the real light source, $I_{\text {smv }}^{\prime}\left(x_{k}, f_{p}, z\right)$ can be obtained as

$$
I^{\prime}{ }_{s m v}\left(x_{k}, f_{p}, z\right)=I_{\text {real }}\left(x_{k}, f_{p}, z\right)
$$

Assuming that the weighting ratio for $k$-th parallax image is $r_{k}, I_{\text {smv }}^{\prime}\left(x_{k}, f_{p}, z\right)$ is given by

$$
I_{s m v}^{\prime}\left(x, f_{p}, z\right)=\sum_{k=1}^{N} I_{s}\left(x-x_{k}\right) \times r_{k} .
$$

Substituting Eq. (7) into Eq. (6), we obtain

$$
I_{S}\left(x_{k}-x_{1}\right) * r_{1}+\cdots+I_{s}\left(x_{k}-x_{N}\right) * r_{N}=I_{\text {real }}\left(x_{k}, f_{p}, z\right) \text {. }
$$

Eq. (8) is an equation with $r_{1} \ldots r_{N}$ as variables and be formulated for all the 1 st to $N$-th parallax image, so it is formulated as the $N$ variables simultaneous equation. Therefore, by obtaining the solutions $r_{1} \ldots r_{N}$ from this equation, it is possible to obtain the optimal combination of weighting ratios for each parallax image to reconstruct the blur image.

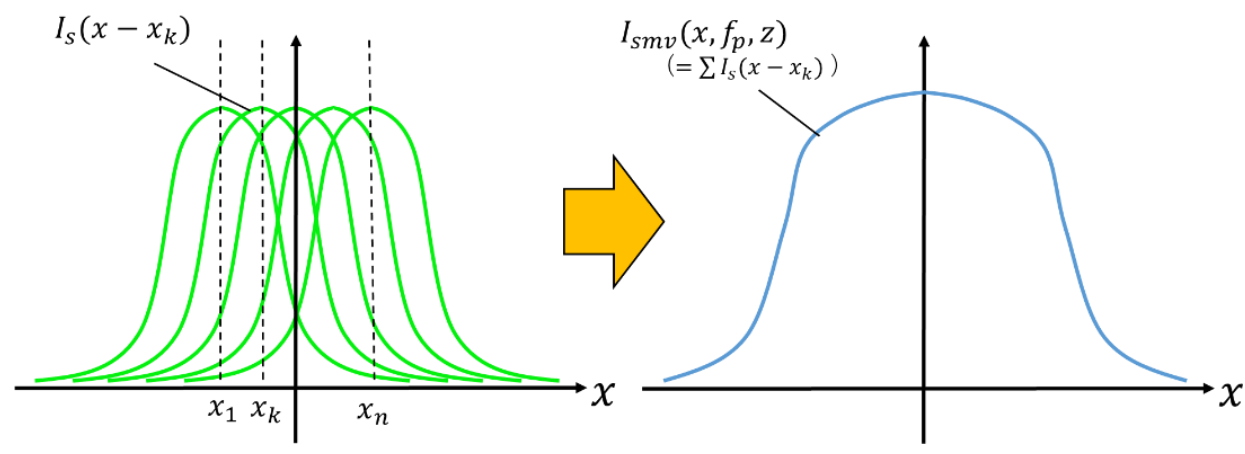

Figure 7. Retinal image reconstructed due to each parallax image. As shown in the left figure, each parallax image is projected to the position $x_{k}$ on the retina, and the sum of them gives the retinal image as shown in the right figure. 


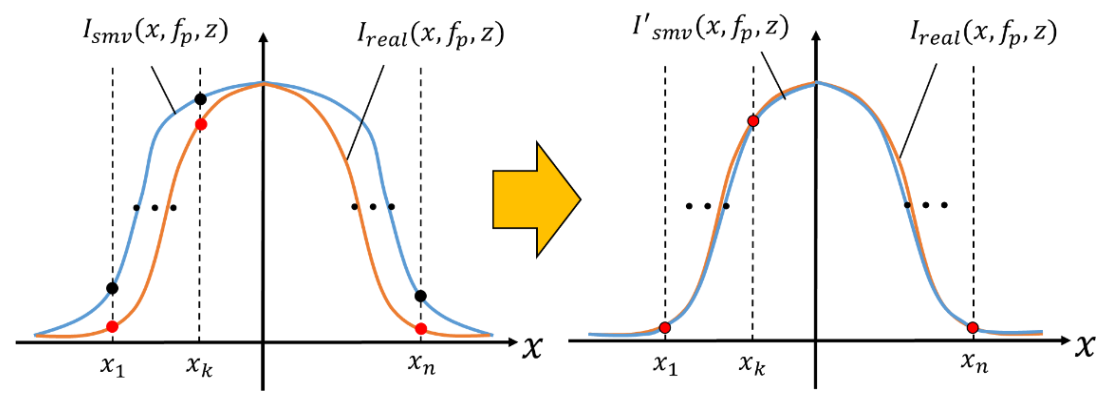

Figure 8. At the position of $x_{k}, I_{\mathrm{smv}}\left(x_{k}, f_{p}, z\right)$ is made equal to $I_{\text {real }}\left(x_{k}, f_{p}, z\right)$ by weighting the light intensity distribution of each parallax image. Therefore, reproducibility of the blur image is improved.

\subsection{Relationship the number of parallax images and the 3D image displayable range}

In a super multi-view 3D HMD, when the observer is not focused on the 3D image, retinal images due to each parallax image shift and overlap on the retina and they recognize the 3D image as the blur image. However, depending on the degree of the overlap of the parallax images, they may recognize not "one $3 \mathrm{D}$ image" but "multiple independent $2 \mathrm{D}$ images". The condition for recognizing a virtual image as "one 3D image" is that the light intensity contrast $m$ between peaks and valleys in the light intensity distribution of the retinal image composed of two adjacent retinal images is less than $10 \%$ as shown in Figure 9. In the light intensity distribution of the composed retinal image, when the peak is $I_{\max }$ and the valley is $I_{\min }, m$ is given as

$$
m=\frac{I_{\max }-I_{\min }}{I_{\max }+I_{\min }} .
$$

Therefore, the displayable range of the 3D image in the proposed method is the display range of virtual image in which the light intensity distribution $I_{\mathrm{smv}}$ of the retinal image reconstructed by the super multi-view method satisfies $m<0.1$ at any position the observer's eye focuses on. $m$ influences the degree of the overlap of parallax images, and as shown in Figure 10, the smaller the shift interval $x_{k+1}-x_{k}$ of parallax images, the smaller $m$. From Eqs. (2), (3) and (4), $x_{k+1}-x_{k}$ is given by

$$
x_{k+1}-x_{k}=\frac{D}{N-1}\left(1-\frac{a-f_{p}}{a f_{p}} L\right) .
$$

From Eq. (10), $x_{k+1}-x_{k}$ depends on $N$, and becomes larger when the position the observer's eye focuses on and the position the image is displayed are more separated. Therefore, in order to enable 3D display in the depth range of human ocular accommodation from $200 \mathrm{~mm}$ to $2000 \mathrm{~mm}$, the number of parallax images is required to exceed the minimum number which satisfies $m<0.1$ when a unit pixel is displayed at $200 \mathrm{~mm}$ depth position and the observer's eye focuses on $2000 \mathrm{~mm}$ depth position (or vice versa).

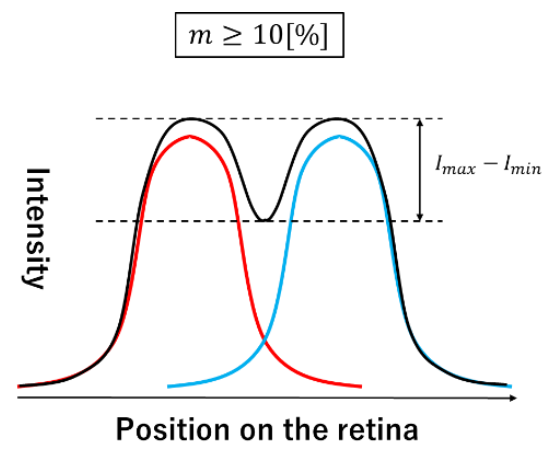

(a)

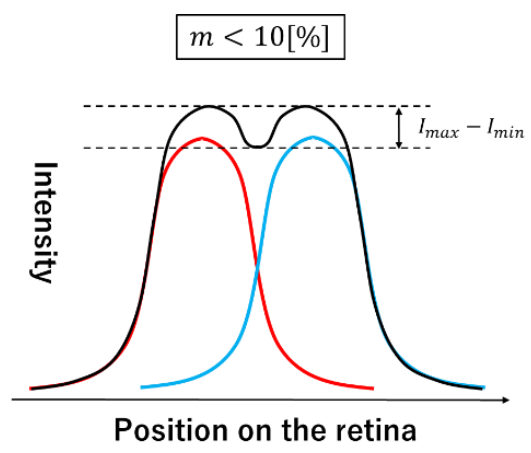

(b)

Figure 9. The light intensity distribution on the retina of two parallax images: (a) recognizing as "one 3D image", (b) recognizing as "multiple independent 2D images" 


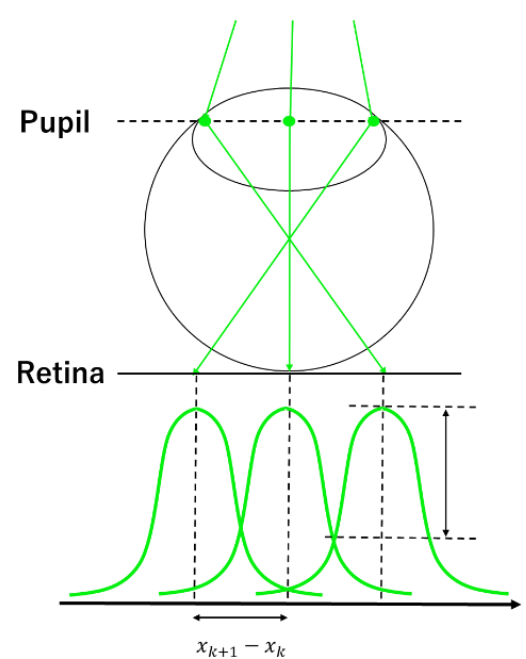

(a)

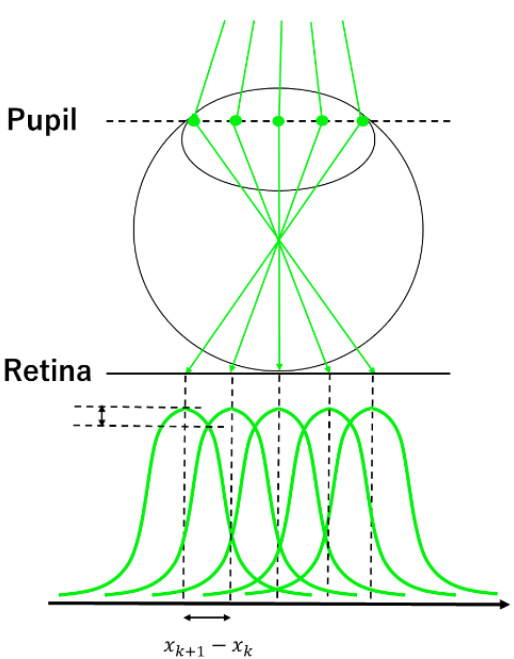

(b)

Figure 10. Comparison of $m$ between the case which $\left(x_{k+1}-x_{k}\right)$ is large and the case which $\left(x_{k+1}-x_{k}\right)$ is small: (a) the case which $\left(x_{k+1}-x_{k}\right)$ is large, (b) the case which $\left(x_{k+1}-x_{k}\right)$ is small.

\section{EXPERIMENTAL RESULTS}

We constructed the prototype system to show the effectiveness of the proposed method described in Chapter 3 and evaluated the shape of the light intensity distribution of the blur image and the 3D displayable range in the method. Figure 11 shows the schematic diagram of the prototype system. In this paper, we did the experiment using a camera instead of the human eye. First, we did an experiment for evaluating the shape of the light intensity distribution of the blur image in the proposed method. The number of parallax images was set 6 , and the camera was focused at $2000 \mathrm{~mm}$ depth position when the vertical line shaped virtual image was displayed at $600 \mathrm{~mm}$ depth position. We compared the results with and without weighting each light intensity distribution of the projected parallax images on the retina based on Section 3.2. Figure 12 (a) (b) show the simulation results of the light intensity distributions $I_{\text {real }}$ and $I_{\text {smv }}$ of the retinal image due to the real source and the prototype system. This simulation is based on the parameters of the prototype system. Table 2 shows the weighting ratio of each parallax image based on Eq. (8). Figures 13 (a) (b) and 14 (a) (b) show images taken by the camera and the light intensity distribution of them with and without weighting each parallax image based on weighting ratios in Table 2. From the results of Figure 12 (a), 14 (a), and (b), it proves that the shape of the light intensity distribution of the blur reconstructed by the prototype system is more similar one of the real blur by weighting, and the reproducibility of the blur is improved in the super multi-view HMD. Furthermore, $m$ obtained from results of Figure 12 (b) and Figure 14 (b) was both $0 \%$. Therefore, it was found that the prototype system based on the proposed method, reconstructed the blur with high reproducibility and enabled 3D display at $600 \mathrm{~mm}$ depth position.

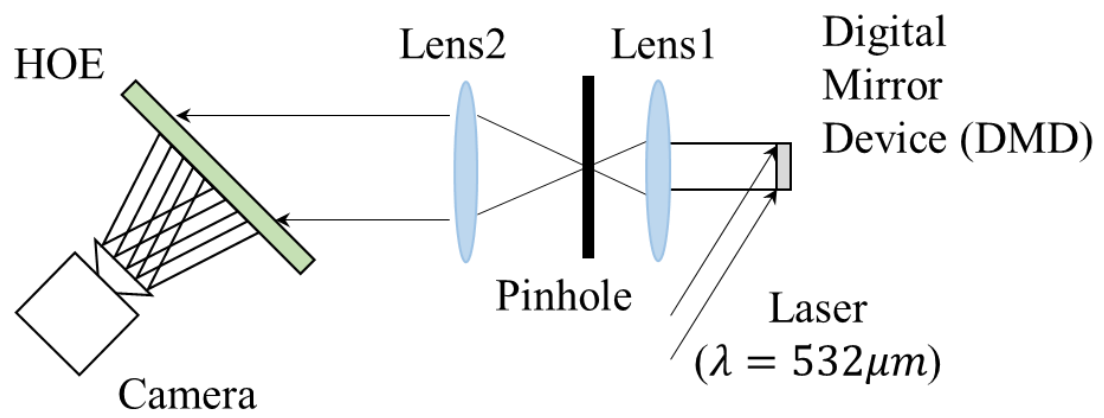

Figure 11. Prototype system. 


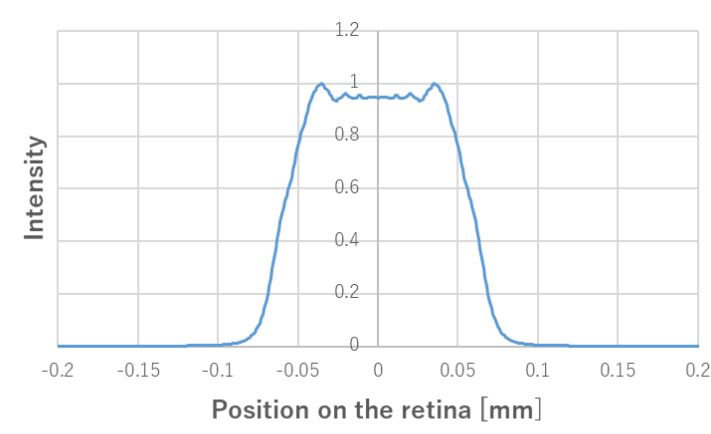

(a)

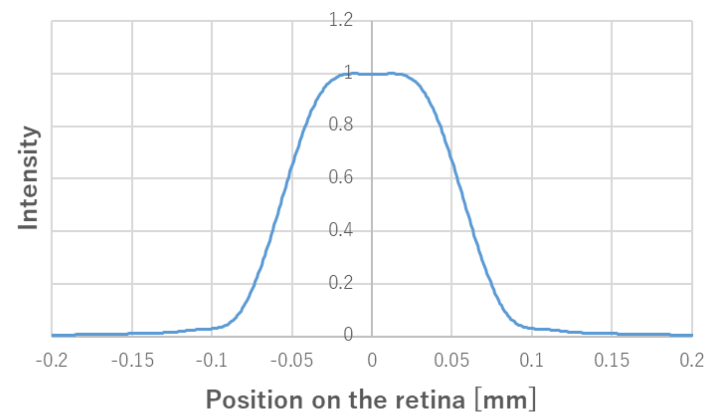

(b)

Figure 12. Simulation results based on analysis: (a) $I_{\text {real }}$ (b) $I_{\text {smv }}$.

Table 2. The weighting ratios of each parallax image.

\begin{tabular}{|c|c|c|c|c|c|}
\hline$r_{1}$ & $r_{2}$ & $r_{3}$ & $r_{4}$ & $r_{5}$ & $r_{6}$ \\
\hline 0.67 & 0.12 & 0.47 & 0.47 & 0.12 & 0.67 \\
\hline
\end{tabular}

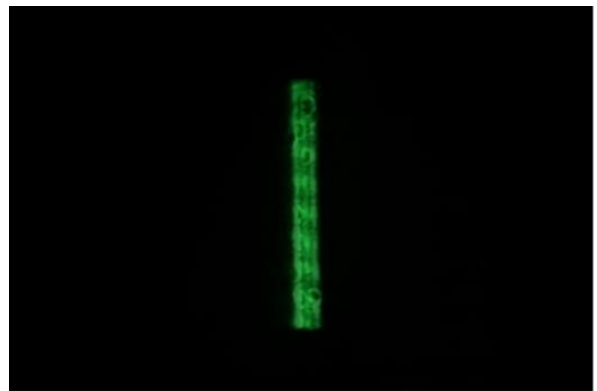

(a)

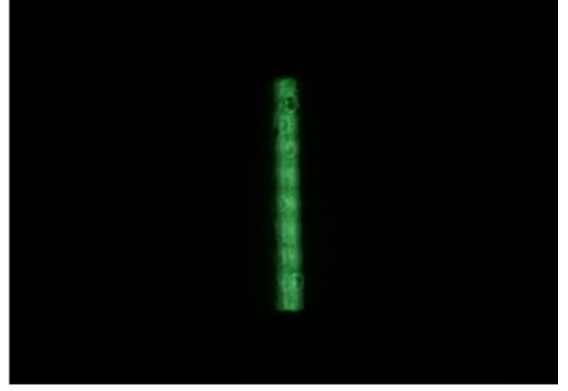

(b)

Figure 13. Images taken by the camera: (a) with weighting each parallax image. (b) without it.

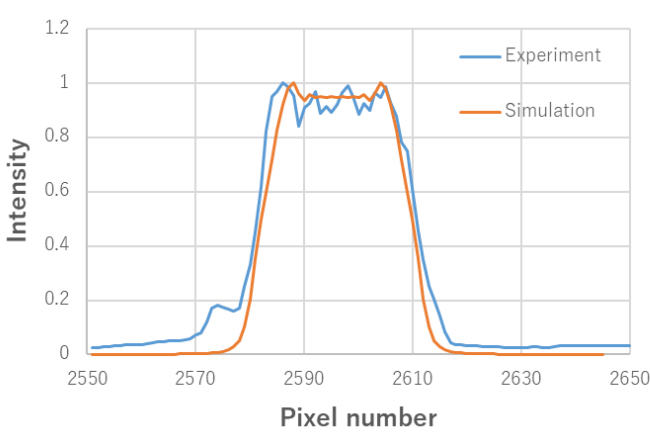

(a)

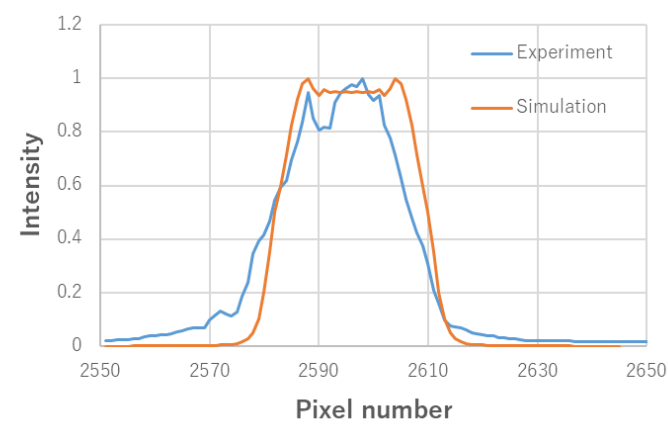

(b)

Figure 14. The light intensity distribution of images taken by the camera: (a) with weighting each parallax image. (b) without it. 
Next, in order to confirm that the prototype system based on the proposed method can display 3D images in the range of $200 \mathrm{~mm}$ to $2000 \mathrm{~mm}$ depth position, the camera was focused at 2000mm depth position and the vertical line shaped virtual image was displayed at $200 \mathrm{~mm}$ depth position. In this situation, Simulation results of $I_{\mathrm{smv}}$ when the number of parallax images is 7 and 8 are shown in Figure 15 (a) and (b). As a result of calculating $m$ from Figure 15 (a) and (b), it was $27.4 \%$ when the number of parallax images was 7 , and $9.1 \%$ when the number of parallax images was 8 . Therefore, it was found that the minimum number of parallax images for which $m$ is less than $10 \%$ is 8 . The light intensity distributions of images taken the camera when the number of parallax images is 7 and 8 are shown in Figure 16 (a) and (b). As a result of calculating $m$ from Figure 16 (a) and (b), it was 26.4\% in Figure 16 (a), and 8.7\% in Figure 16 (b). Therefore, it was found that at least 8 parallax images were required to enable display 3D image in the range of $200 \mathrm{~mm}$ to $2000 \mathrm{~mm}$ depth position in the prototype system. From the above results, the effectiveness of the proposed method is shown.

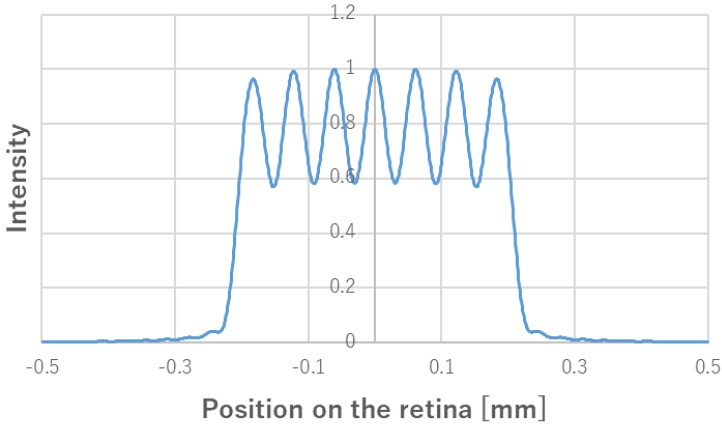

(a)

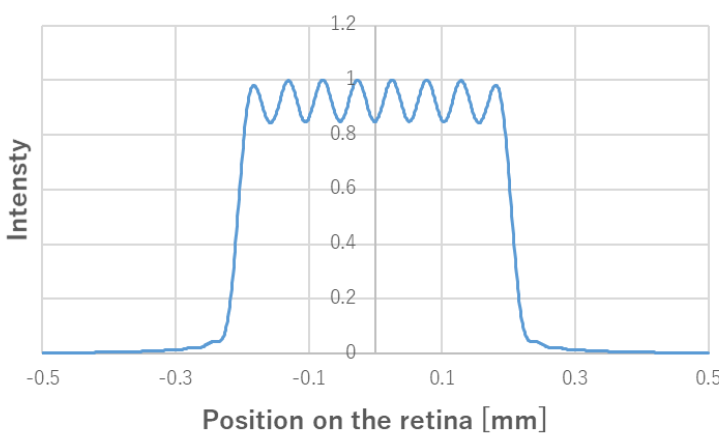

(b)

Figure 15. Simulation results of $I_{\mathrm{smv}}$ when the position of the virtual image is $200 \mathrm{~mm}$ depth position and the position at which the camera is focused is $2000 \mathrm{~mm}$ depth position: (a)when the number of parallax images is 7 . (b) when the number of parallax images is 8 .

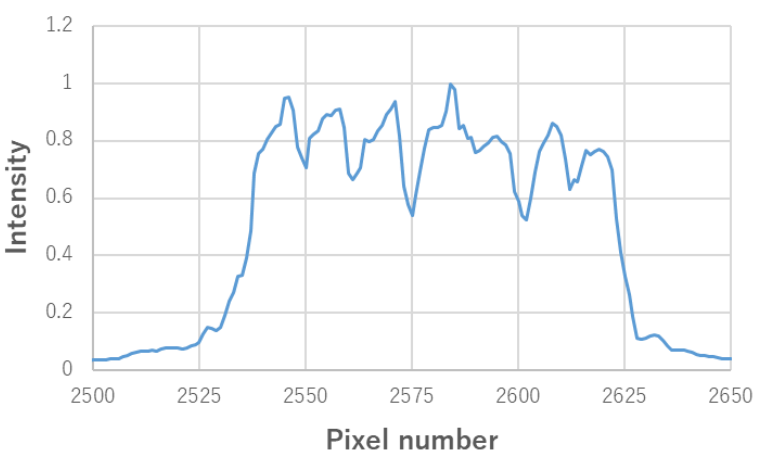

(a)

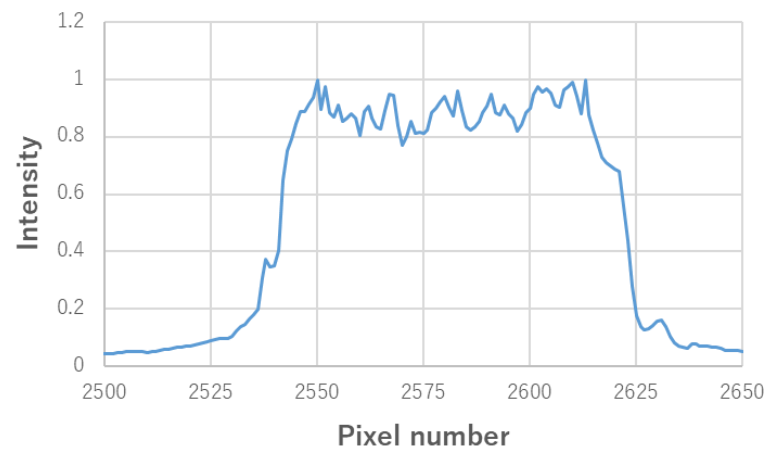

(b)

Figure 16. The light intensity distribution of images taken by the camera when the position of the virtual image is $200 \mathrm{~mm}$ depth position and the position at which the camera is focused is $2000 \mathrm{~mm}$ depth position: (a)when the number of parallax images is 7 . (b) when the number of parallax images is 8 . 


\section{CONCLUSION}

In this paper, we verified the relationship between the number and the light intensity of the parallax images and the light intensity distribution of the retinal image and proposed the optimal method so that the blur image reconstructed by the retinal projection type super multi-view HMD is more similar a real blur. We constructed the prototype system and evaluated the proposed method. From the experimental results, it was confirmed that the reproducibility of the blur image reconstructed by the prototype system was improved by weighting the light intensity of the projected parallax images on the retina. Furthermore, we confirmed that when the number of parallax images was 8 or more, the prototype system can display 3D images in the range of $200 \mathrm{~mm}$ to $2000 \mathrm{~mm}$ depth position. Therefore, the proposed retinal projection type super multi-view HMD can display more natural 3D images in the depth range of human ocular accommodation from $200 \mathrm{~mm}$ to $2000 \mathrm{~mm}$ by confirming the relationship among the number of parallax images, the light intensity of parallax images and the light intensity distribution of the retinal image.

\section{ACKNOWLEDGEMENT}

This work was partially supported by JSPS KAKENHI Grant Number $18 \mathrm{H} 03277$.

\section{REFERENCES}

[1] Tamura, H. and Ohta, V., [Mixed Reality], Ohmsha (1999).

[2] Takahashi, H., Ito, Y., Nakata, S. and Yamada, Y., "Retinal projection type super multi-view head-mounted display," Proc. SPIE-IS\&T Electronic Imaging, 9012, 90120L1-9012L6 (2014).

[3] Ito, Y., Takaichi, K. and Takahashi, H., "See-Through Retinal Projection Head-Mounted Display by using the Extended Maxwellian View," ICIC Express Letters 7(6), 1755-1760 (2013).

[4] Takatsuka, Y., Yoshimoto, K. and Takahashi, H., "Retinal projection type 3D head-mounted display using an HOE lens array," Proc. SPIE-IS\&T Electronic Imaging 2016, SDA-428.1-SDA-428.6 (2016).

[5] Emoto, T., Konda, T., Yoshimoto, K. and Takahashi, H., "Analysis of retinal images for retinal projection type super multi-view 3D head-mounted display," Proc. IS\&T Electronic Imaging 2017, 188-193 (2017).

[6] Konda, T., Tanaka, K., Yoshimoto, K. and Takahashi, H., "Retinal projection type super multi-view 3D headmounted display using the time division projection optical system," Proc. SPIE 10556, 105560L1-105560L11 (2018).

[7] Maeda, H., Kohno, J., Yoshimoto, K. and Takahashi, H., "Analysis of displayable depth range for retinal projection type super multi-view 3D head-mounted display using the time division projection optical system," Proc. SPIE 10942, Advances in Display Technologies IX, 109420X (2019).

[8] Kajiki, Y., Yoshikawa, H. and Honda, T., "Ocular Accommodation by Super multi-view Stereogram and 45 View Stereoscopic Display," Proc. IDW'96, 2, 489-492 (1996).

[9] Westheimer, G., "The Maxwellian View," Vision Res. 6, 669-682 (1966). 